\title{
A Comparison of Three Regions of Puppis A
}

\author{
K.F. Fischbach, L.M. Bateman, C.R. Canizares, T.H. Markert, and P.J. Saez \\ Massachusetts Institute of Technology, Cambridge, Massachusetts, U.S.A.
}

ABSTRACT High resolution X-ray spectral observations of Puppis A were performed with the FPCS on the Einstein Observatory at three regions of the remnant: the shock front, the bright eastern knot, and the interior. Plasma diagnostics of lines from OVII and OVIII constrain the values of electron temperature, ionization timescale, and hydrogen column density. We compare results of the diagnostics for these three regions. A nonequilibrium analysis of previously published fluxes of oxygen lines shows that the interior has not yet reached ionization equilibrium.

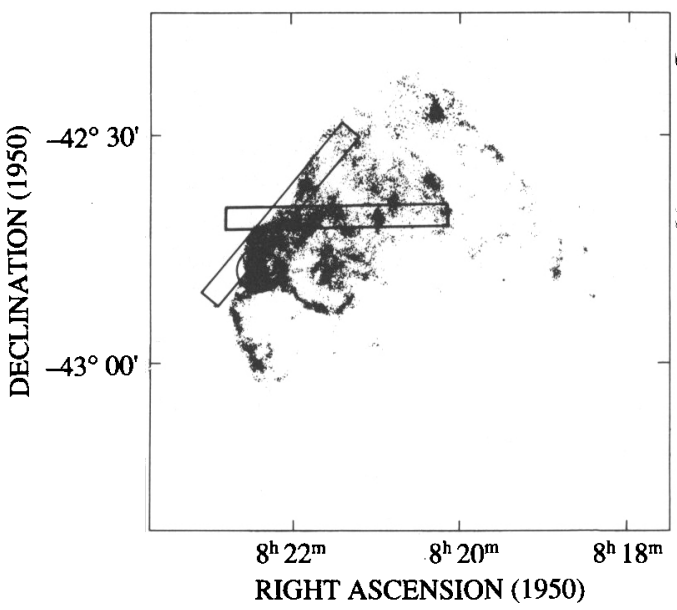

Figure 1 Aperture positions for the bright eastern knot, the shock front and the interior overlaid on an HRI image of Petre, et al. (1981).
Between April, 1979 and July, 1980, the Puppis A supernova remnant was observed using the Focal Plane Crystal Spectrometer (FPCS) on the Einstein Observatory (Canizares, et al. 1979). The remnant was observed at several positions; the bright eastern knot, the shock front, and at several closely spaced positions in the interior. A circular (6 arc minute) aperture was used to observe the bright eastern knot; in the other cases a $3 \times 30$ arc minute rectangular aperture was used to make the observations. The interior was observed with the rectangular aperture in a variety of angular orientations. Figure 1 shows FPCS aperture positions for the three regions, overlaid on the Puppis A image obtained by Petre et al. (1981). The X-ray observations consisted primarily of lines of helium-like and hydrogen-like ions of oxygen (O VII and $\mathrm{O}$ VIII) and neon ( $\mathrm{Ne}$ IX and $\mathrm{Ne} \mathrm{X}$ ), and neon-like iron (Fe XVII).

The X-ray emission lines detected by the FPCS provide useful diagnostics of conditions in the line-emitting plasma. For transitions $i \rightarrow g$ and $j \rightarrow k$ of ionization states $+x$ and $+y$ of an element $\mathrm{Z}$, the ratio of $\mathrm{X}$-ray fluxes is given by:

$$
\frac{f_{i g}}{f_{j k}}=\frac{\Omega_{i g} \times n_{+x} \times \exp \left(-\sigma\left(E_{i g}\right) N_{H}\right) \times \exp \left(-E_{i g} /\left(k T_{e}\right)\right)}{\Omega_{j k} \times n_{+y} \times \exp \left(-\sigma\left(E_{j k}\right) N_{H}\right) \times \exp \left(-E_{j k} /\left(k T_{e}\right)\right)}
$$

where $\Omega_{\mathrm{ig}}$ is the effective collision strength for transition $\mathrm{i} \rightarrow \mathrm{g}, \mathrm{E}_{\mathrm{ig}}$ is the excitation energy, $T_{e}$ the electron temperature, $n_{+x}$ the density of ion $x, \sigma$ the cross-section per hydrogen atom for photoelectric absorption at energy $\mathrm{E}_{\mathrm{ig}}$, and $\mathrm{N}_{\mathrm{H}}$ the hydrogen column 
density (see Vedder et al. 1986). Since it is difficult to make enough measurements so as to solve for all of the unknowns, the approach we have taken is to measure a few line intensities selected so that as many parameters as possible will cancel. For example, two lines from the same ion define an allowed region in the parameter space of column density $\mathrm{N}_{\mathrm{H}}$ and electron temperature $\mathrm{T}_{\mathrm{e}}$. In order to use two different ions from the same atom, it is necessary to perform a non-equilibrium analysis because the relative abundances of the various ions are functions of time since the plasma was shocked. The ionization structure is determined by solving a set of $\mathrm{Z}+1$ simultaneous differential equations. (We have employed the technique of Hughes and Helfand (1985) to solve the ion balance equations.) The resulting ion abundances are then used explicitly in the equations for the line emissivities. The various parameters of the non-equilibrium model are $T_{e}, N_{H}$, and $\tau$. Here $\tau$ ( $\equiv$ electron density $x$ time since the shock) is the ionization timescale and measures the extent to which ionization equilibrium has been attained. The complete analysis technique is discussed in more detail in Markert et al. (1988).

In order to compare the physical conditions of the three regions, (i.e. interior, bright eastern knot and shock front) we compared the results of the analysis described above (Winkler et al. 1981; Winkler et al. 1983; Fischbach et al. 1988) The derived allowable regions in $\left(\mathrm{N}_{\mathrm{H}}, \mathrm{T}_{e}\right)$ and $\left(\mathrm{T}_{e}, \tau\right)$ parameter space are reproduced in Figures 2,3 and 4 for the respective regions. In order to obtain an estimate of the ionization timescale, $\tau$, of the interior, previously published oxygen line fluxes (Winkler et al. 1981) were applied to the non-equilibrium analysis, from which Figure $2 \mathrm{~b}$ is obtained. In the analysis $\mathrm{N}_{\mathrm{H}}$ was assumed to be $4 \times 10^{21} \mathrm{~cm}^{-2}$, but the results are not particularly sensitive to this assumption.

The deduced constraints on column density $\mathrm{N}_{\mathrm{H}}$, electron temperature $T_{e}$, and ionization timescale $\tau$ are shown in Table 1 for the three regions.

\section{TABLE 1}

\section{PLASMA DIAGNOSTICS}

$\begin{array}{lllll}\text { Region } & \mathrm{T}\left(10^{6} \mathrm{~K}\right) & \tau\left(\mathrm{yr} \mathrm{cm}^{-3}\right) & \mathrm{t}(\mathrm{yr})^{(1)} & \mathrm{n}_{\mathrm{e}}\left(\mathrm{cm}^{-3}\right)^{(2)} \\ & & 300-2000 & 3700 & 0.1-0.6 \\ \text { Interior } & 4.3-19^{(3)} & 800-1200 & 100 & 8-12 \\ \text { Eastern Knot } & 6-8 & 150-400 & 100 & 1.5-4\end{array}$

(1) See Winkler et al., 1988; Winkler et al., 1983; Fischbach et al., 1988).

(2) Electron density $n_{e}$ is estimated by naively assuming $\tau=n_{e} t$. In fact, the line-emitting region consists of material shocked at different times, so the reported value of $n_{e}$ is probably a slight underestimate.

(3) Winkler (1981) derives $T_{e} \geq 1.5 \times 10^{6}$. These temperature constraints are obtained from the analysis shown in Figure $2 b$.

The non-equilibrium analysis on oxygen lines from the interior suggests that the plasma departs substantially from ionization equilibrium. Thus, none of the three regions is found to be in ionization equilibrium despite the advanced age ( 3700 years) of the remnant. 
Acknowledgements We thank Jack Hughes for supplying his computer code for the non-equilibrium analysis. We are grateful to Elaine Aufiero for preparing the manuscript. This work was supported in part by NASA grant NAG 8-494.

\section{REFERENCES}

Canizares, C.R., Clark, G.W., Markert, T.H., Berg, C., Smedira, M., Bardas, D., Schnopper, H., and Kalata, K. 1979, Ap. J. (Letters), 234, L33.

Fischbach, K.F., Canizares, C.R., Markert, T.H., and Coyne, J.M. 1988, in Supernova Remnants and the Interstellar Medium, eds. R.S. Roger and T.L. Landecker, (Cambridge University Press, Cambridge) 153.

Hughes, J.P. and Helfand, D.J. 1985, Ap. J., 291, 544.

Markert, T.H., Blizzard, P.L., Canizares, C.R. and Hughes, J.P. 1988, in Supernova Remnants and the Interstellar Medium, eds. R.S. Roger and T.L. Landecker, (Cambridge University Press, Cambridge) 129.

Petre, R., Canizares, C.R., Kriss, G.A. and Winkler, P.F. 1981,Ap. J.,258, 22.

Vedder, P.W., Canizares, C.R., Markert, T.H. and Pradhan, A.K. 1986, Ap. J., 307, 269.

Winkler, P.F., Canizares, C.R., Clark, G.W., Markert, T.H., Petre, R. 1981, Ap. J., 245, 574.

Winkler, P.F., Canizares, C.R. and Bromley, B.C. 1983, in Supernova Remnants and Their X-Ray Emission, eds. J. Danziger and P. Gorenstein, (Dordrecht: Reidel), 245.

Winkler, P.F., Tuttle, J.H., Kirshner, R.P. and Irwin, M.J. 1988, in Supernova Remnants and the Interstellar Medium, eds. R.S. Roger and T.L. Landecker, (Cambridge University Press, Cambridge 65.\}
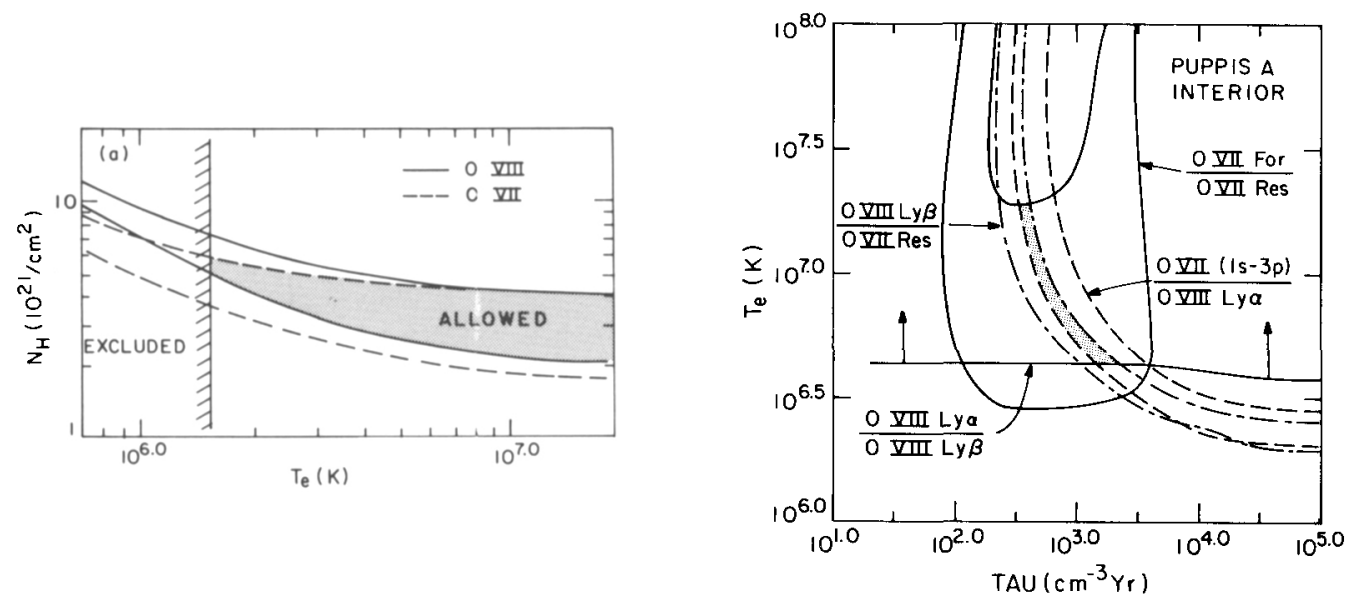

Figures $2 a, b$ Regions in $\left(N_{\mathrm{H}}, T_{\mathrm{e}}\right)$ and $\left(T_{\mathrm{e}}, \tau\right)$ parameter space allowed by FPCS measurements of the interior. Shaded area is region of overlap consistent with all measurements. Figure $2 a$ is reproduced from Winkler et al. (1981). Figure $2 b$ shows results of a non-equilibrium analysis using oxygen lines as published in Winkler et al. (1981) and assuming $N_{\mathrm{H}}=4 \times 10^{21} \mathrm{~cm}^{-2}$. Each pair of contours in Figure $2 b$ indicates $90 \%$ confidence. 

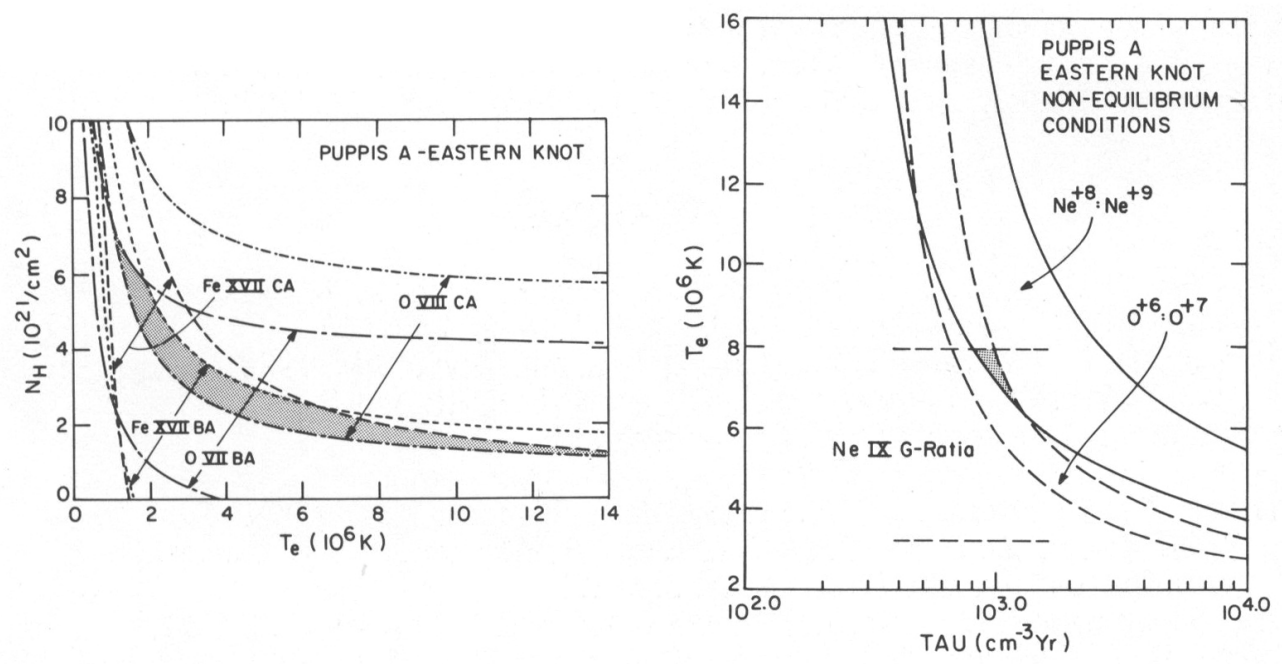

Figures $3 a, b$ Allowed (shaded) regions in $\left(N_{\mathrm{H}}, T_{\mathrm{e}}\right)$ and $\left(T_{\mathrm{e}}, \tau\right)$ parameter space for the bright eastern knot (Winkler et al. 1983).
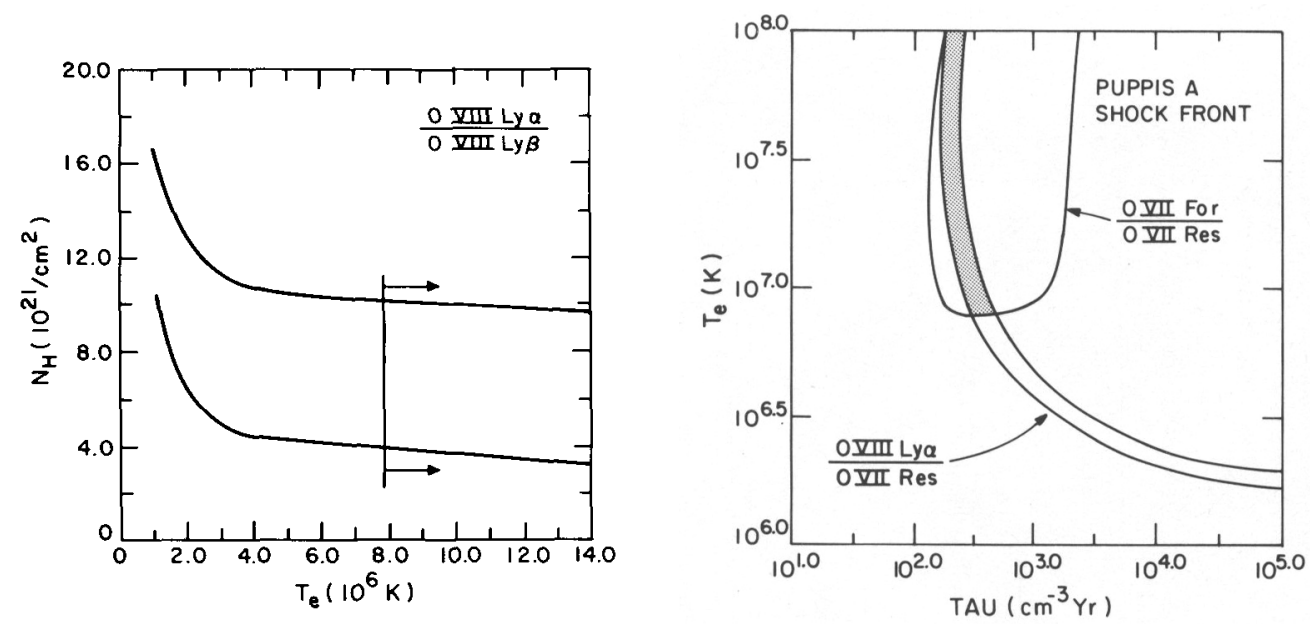

Figures 4a,b Allowed (shaded) regions in $\left(N_{\mathrm{H}}, T_{\mathrm{e}}\right)$ and $\left(T_{\mathrm{e}}, \tau\right)$ parameter space for the shock front (Fischbach et al. 1988). Arrows to the right of the vertical line in Figure $3 a$ indicate allowed region. 